\title{
Charge Condensation and Lattice Coupling Drives Stripe Formation in Nickelates
}

\author{
Y. Shen, ${ }^{1, *}$ G. Fabbris $\odot,{ }^{1,2}$ H. Miao $\odot,{ }^{1,3}$ Y. Cao, ${ }^{1,4}$ D. Meyers, ${ }^{1,5}$ D. G. Mazzone $\odot,{ }^{1,6}$ T. Assefa $\odot,{ }^{1}$ X. M. Chen, ${ }^{1}$ \\ K. Kisslinger, ${ }^{7}$ D. Prabhakaran, ${ }^{8}$ A. T. Boothroyd, ${ }^{8}$ J. M. Tranquada $\odot,{ }^{1}$ W. Hu, ${ }^{9}$ A. M. Barbour, ${ }^{9}$ S. B. Wilkins, ${ }^{9}$ \\ C. Mazzoli, ${ }^{9}$ I. K. Robinson, ${ }^{1}$ and M. P. M. Dean $\oplus^{1, \dagger}$ \\ ${ }^{1}$ Condensed Matter Physics and Materials Science Department, Brookhaven National Laboratory, Upton, New York 11973, USA \\ ${ }^{2}$ Advanced Photon Source, Argonne National Laboratory, Lemont, Illinois 60439, USA \\ ${ }^{3}$ Material Science and Technology Division, Oak Ridge National Laboratory, Oak Ridge, Tennessee 37830, USA \\ ${ }^{4}$ Materials Science Division, Argonne National Laboratory, Lemont, Illinois 60439, USA \\ ${ }^{5}$ Department of Physics, Oklahoma State University, Stillwater, Oklahoma 74078, USA \\ ${ }^{6}$ Laboratory for Neutron Scattering and Imaging, Paul Scherrer Institut, CH-5232 Villigen, Switzerland \\ ${ }^{7}$ Center for Functional Nanomaterials, Brookhaven National Laboratory, Upton, New York 11973, USA \\ ${ }^{8}$ Department of Physics, University of Oxford, Clarendon Laboratory, Oxford OXI 3PU, United Kingdom \\ ${ }^{9}$ National Synchrotron Light Source II, Brookhaven National Laboratory, Upton, New York 11973, USA
}

(Received 27 October 2020; accepted 31 March 2021; published 30 April 2021)

\begin{abstract}
Revealing the predominant driving force behind symmetry breaking in correlated materials is sometimes a formidable task due to the intertwined nature of different degrees of freedom. This is the case for $\mathrm{La}_{2-x} \mathrm{Sr}_{x} \mathrm{NiO}_{4+\delta}$, in which coupled incommensurate charge and spin stripes form at low temperatures. Here, we use resonant $\mathrm{x}$-ray photon correlation spectroscopy to study the temporal stability and domain memory of the charge and spin stripes in $\mathrm{La}_{2-x} \mathrm{Sr}_{x} \mathrm{NiO}_{4+\delta}$. Although spin stripes are more spatially correlated, charge stripes maintain a better temporal stability against temperature change. More intriguingly, charge order shows robust domain memory with thermal cycling up to $250 \mathrm{~K}$, far above the ordering temperature. These results demonstrate the pinning of charge stripes to the lattice and that charge condensation is the predominant factor in the formation of stripe orders in nickelates.
\end{abstract}

DOI: 10.1103/PhysRevLett.126.177601

Emergent phenomena in strongly correlated materials arise due to multifarious interactions among charge, spin, and lattice degrees of freedom. Such complexity hampers the ability to understand their remarkable states and realize new functionalities [1]. Identifying dominant interaction is, however, challenging, as different interactions act simultaneously and can yield complex ground states with more than one form of order [2]. A representative phenomenon of this type is the electronic stripes that appear in various strongly correlated materials [3-6]. These effects have been considered extensively in cuprate high-temperature superconductors, which host charge and sometimes spin stripe order, typically with a simple factor-of-2 relationship between the charge and spin incommensurabilities [7-9]. Nickelates also host both superconductivity and stripe order [10-12], but no system has yet been shown to simultaneously host both orders. The existence of stripe order in $\mathrm{La}_{4} \mathrm{Ni}_{3} \mathrm{O}_{8}$, which appears rather similar to superconducting $\mathrm{Nd}_{1-x} \mathrm{Sr}_{x} \mathrm{NiO}_{2}$ [13-16], does, however, support the likely proximity of stripe order and superconductivity. While static stripe order appears to suppress bulk 3D superconductivity, some researchers have suggested that stripe fluctuations may act to promote superconductivity [17-19]. Therefore, understanding the driving forces behind charge and spin stripe formation and dynamics in strongly correlated materials has attracted considerable attention and may be crucial to understanding unconventional superconductivity. Stripe formation has been studied in the past through detailed measurements of stripe transition temperatures and correlation lengths [20-28] and associated Landau model analysis [29,30]. The problem has also been addressed via model Hamiltonian analysis that suggested that lattice coupling might be crucial to stabilize stripes [31,32]. The implementation of resonant $\mathrm{x}$-ray photon correlation spectroscopy (XPCS) at modern lowemittance synchrotron sources opens new routes to directly probe stripe formation and dynamics [33-36].

Herein, we report the first resonant XPCS experiment to simultaneously probe charge order (CO), spin order (SO), and lattice coupling in a stripe-ordered material, focusing on the prototypical material $\mathrm{La}_{2-x} \mathrm{Sr}_{x} \mathrm{NiO}_{4+\delta}$ (LSNO) with $x=0.225$ and $\delta=0.07$. Although SO is more correlated and stable at $70 \mathrm{~K}, \mathrm{CO}$ is more robust in temporal stability against temperature changes, which we attribute to electron-phonon coupling (EPC). This is further supported by our discovery that the $\mathrm{CO}$ domains are effectively pinned to the lattice and the corresponding speckle patterns remain highly reproducible with thermal cycling up to $250 \mathrm{~K}$, well above the transition temperature $T_{\mathrm{CO}}$. $\mathrm{SO}$, however, is not directly coupled to the lattice and loses its domain memory 
once the sample is warmed across the magnetic transition temperature $T_{\mathrm{SO}}$. These results imply that charge condensation, and its coupling to the lattice and disorder, is the driving force behind stripe ordering.

X-ray measurements were carried out at the Coherent Soft X-Ray (CSX) 23-ID-1 beam line at the National Syncrotron Light Source II with $\mathrm{x}$-ray energy tuned to the $\mathrm{Ni} L_{3}$ edge [Fig. 1(a)]. The LSNO single crystal was synthesized by the

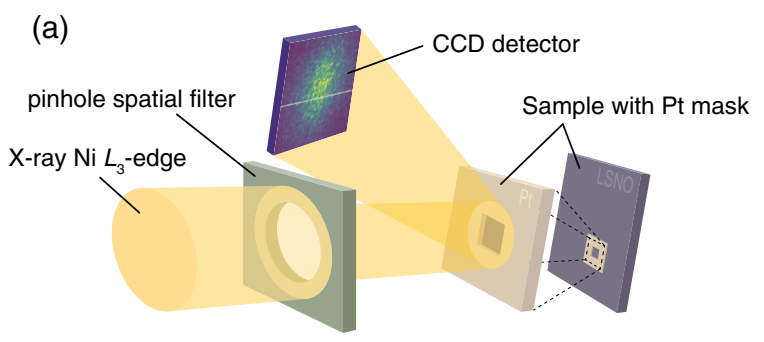

(b)

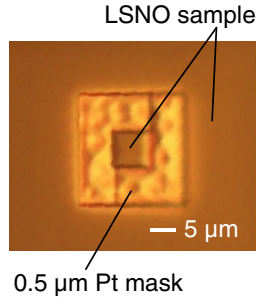

(d)

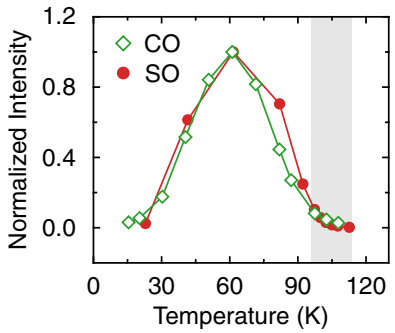

(c)

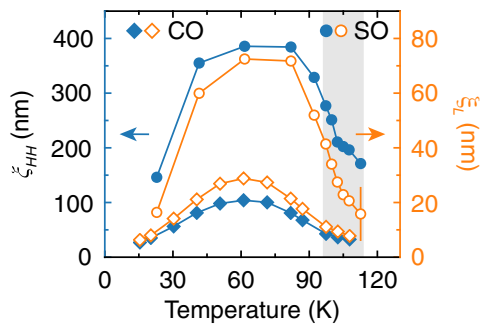

(e)

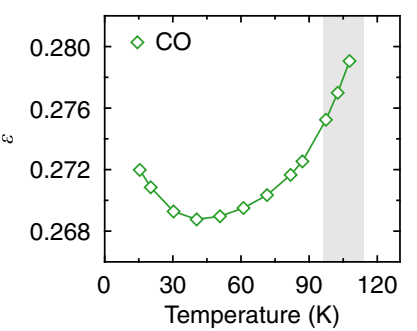

FIG. 1. Experimental configuration and $\mathrm{CO}$ and $\mathrm{SO}$ superlattice peaks. (a) The instrumental setup for the measurements at CSX. The x-ray beam is set to the $\mathrm{Ni} L_{3}$-edge energy and tuned in order to maximize the strength of the $\mathrm{CO}$ and SO intensity [39]. It then propagates through the pinhole and is scattered by the LSNO sample onto the detector. For the domain memory study, a $0.5-\mu \mathrm{m}$-thick Pt mask was deposited on the sample [39]. (b) An optical micrograph of the Pt mask on the (110) surface of a LSNO single crystal. (c) Temperature dependence of the correlation lengths along $[H, H, 0]$ and $[0,0, L]$ directions. The correlation length is defined as $\xi=d /$ HWHM, where HWHM stands for half width at half maximum in reciprocal lattice units and $d$ is the unit cell size in the appropriate direction [44]. (d) Temperature dependence of the peak heights evaluated from fitting of the $\mathrm{CO}$ and $\mathrm{SO}$ superlattice peaks, which are normalized according to their values at $60 \mathrm{~K}$. The signals were fitted with a three-dimensional Lorentzian function. (e) Incommensurability defined by the peak position of the $\mathrm{CO} Q$ vector as a function of the temperature. The shaded areas indicate the onset temperature range for $\mathrm{CO}$ and $\mathrm{SO}$. floating-zone method with a Sr concentration of $x=0.225$ [37]. As shown later, the $\mathrm{CO}$ incommensurability is $\epsilon \approx 0.27$, larger than $x$, which is likely related to oxygen doping, since $\delta=0.07$ [38]. The sample's surface normal was close to the $[H, H, 0]$ direction. Thus, we made $(H, H, L)$ the scattering plane and focused on peaks with $\boldsymbol{Q}_{\mathrm{CO}}=(\epsilon, \epsilon, 1)$ and $\boldsymbol{Q}_{\mathrm{SO}}=(1 / 2-\epsilon / 2,1 / 2-\epsilon / 2,0)$ [39]. The reciprocal lattice units (r.l.u.) is defined in terms of $\boldsymbol{Q}=$ $(H, K, L)=(2 \pi / a, 2 \pi / b, 2 \pi / c)$ within the space group I4/ $\mathrm{mmm}$ and $a=b=3.84 \AA$ and $c=12.65 \AA$. For the domain memory measurements, we used a $0.5-\mu \mathrm{m}$-thick Pt mask, which had been deposited on the sample in order to reproducibly illuminate the same sample volume independent of possible thermal drifts in the sample position [Fig. 1(b)] [39].

We start by characterizing the superlattice peaks corresponding to $\mathrm{CO}$ and $\mathrm{SO}$ at different temperatures using standard resonant $\mathrm{x}$-ray diffraction. With decreasing temperature, the peak heights first increase substantially through the transition temperatures along with enhanced correlation lengths for both $\mathrm{CO}$ and SO [Figs. 1(c) and 1(d)]. Below $\sim 70 \mathrm{~K}$, the peak heights drop and the spatial correlations are relaxed, consistent with previous reports $[26,45,46]$. The reason for this is not uniquely determined, but it may be connected to a spin reorientation at lower temperature [27] or the influence of spin exchange interactions [26]. Throughout the temperature range, the correlation lengths along the $[H, H, 0]$ direction are much larger than those along $[0,0, L]$ and $\mathrm{SO}$ possesses a larger correlation length than $\mathrm{CO}$ [Fig. 1(c)]. Because of the critical fluctuations and shortrange correlations near the phase transitions, the onset temperatures $T_{\mathrm{CO}}$ and $T_{\mathrm{SO}}$ are not uniquely defined. We estimate them both to occur between 96 and $114 \mathrm{~K}$. Regarding the incommensurability, the intersite Coulomb repulsion tends to stabilize $\epsilon$ equal to the hole concentration [47], while the commensurability effect optimizes stripe formation at $x=1 / 3$. The actual incommensurability is a compromise of these two factors [23]. With increasing temperature, thermal fluctuations are expected to start to outcompete Coulomb repulsion [45,48,49], driving the incommensurability closer to $1 / 3$ at higher temperature [Fig. 1(e)].

To elucidate the temporal stabilities of $\mathrm{CO}$ and $\mathrm{SO}$, we employ XPCS to study the domain distribution and its fluctuations. In XPCS, the coherent photons scattered by different domains interfere with each other, leading to a complex "speckle" pattern modulated by the usual diffraction line shape [33,34,36,50-52]. Figures 2(a) and 2(b) show the representative speckles of the $\mathrm{CO}$ and $\mathrm{SO}$ superlattice peaks at $70 \mathrm{~K}$. The shape of the peak envelope is determined by the spatial correlations and instrument geometry. In particular, the horizontal width of the SO peak is mainly determined by the correlations along the $[-1,1,0]$ direction, while the vertical width is dominated by $c$-axis correlations, elongating the envelope vertically. For the $\mathrm{CO}$ peak, the 


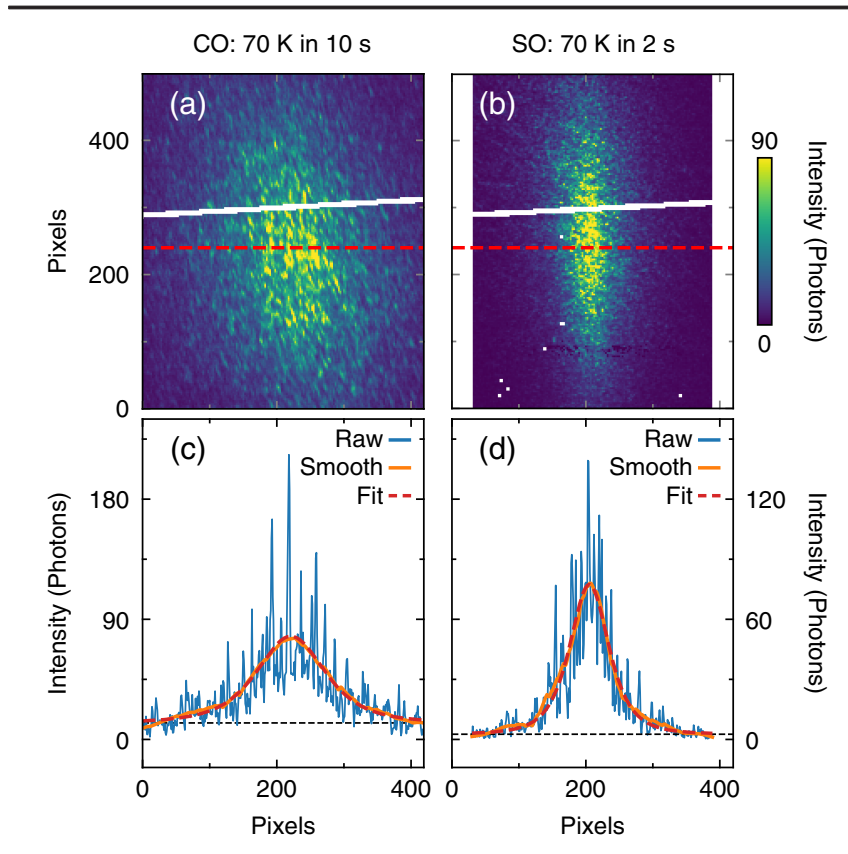

FIG. 2. Speckle patterns of CO and SO. (a),(b) Representative detector images around the $\mathrm{CO}$ and $\mathrm{SO}$ superlattice peaks measured with a $10 \mu \mathrm{m}$ pinhole. The white pixels arise from the beamstop or detector errors and are omitted from the data. (c), (d) Line cuts through the horizontal red dashed lines in (a) and (b). The envelope of the peak is estimated by smoothing and fitting processes that are shown as red and orange lines, respectively. The black dashed lines are uniform fluorescent background evaluated from fittings.

vertical width has less contribution from $c$-axis correlations so that the envelope appears more isotropic. Meanwhile, the distribution of the underlying stripe domains is encoded in the positions of the speckles [35], and the shape of the speckles is determined by the Fourier transform of the beam footprint projected onto the detector. The nonzero $L$ component of the $\mathrm{CO}$ peak makes the footprint of the beam more anisotropic. To show the speckle modulation more clearly, we present in Figs. 2(c) and 2(d) the line cuts through the red dashed lines in Figs. 2(a) and 2(b). The peak envelope is estimated by two independent methods: smoothing with the Savitzky-Golay filter and fitting with a squared Lorentzian function. The sharp speckle modulation observed here indicates that the fluctuations for $\mathrm{CO}$ and $\mathrm{SO}$ are slower than the time window of the measurements, which is $1 \mathrm{~s}$ at $70 \mathrm{~K}$ [39]. Otherwise, the contrast of the interference patterns will be significantly reduced [33].

In order to quantify the fluctuation timescale, we measure the time dependence of the speckle patterns and calculate the normalized one-time correlation function [33]

$$
g_{2}(\tau)=\frac{\langle I(t) I(t+\tau)\rangle}{\langle I(t)\rangle^{2}}=1+\beta|F(\tau)|^{2},
$$
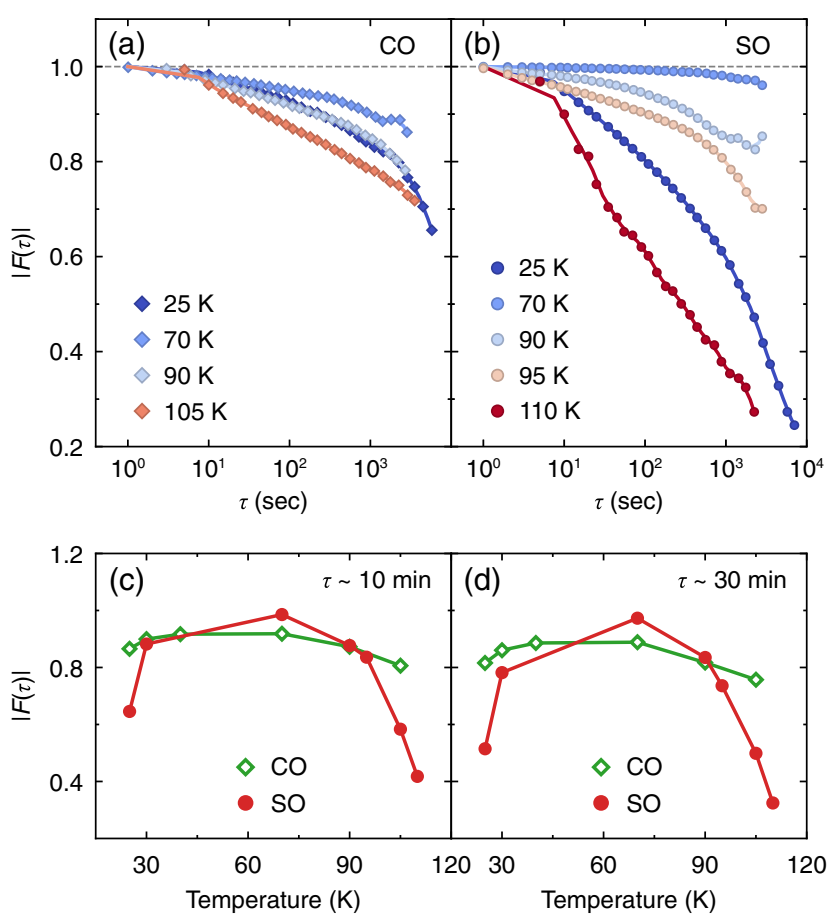

FIG. 3. Temporal stability of $\mathrm{CO}$ and SO. (a),(b) Time dependence of the intermediate scattering functions at different temperatures. The solid lines are guides to the eye. (c),(d) The scattering functions after certain time delays.

where $I$ represents the total intensity including background, $\tau$ is the lag time, and $\langle\cdots\rangle$ stands for the time and ensemble average. The time-dependent evolution can be extracted from the intermediate scattering function $|F(\tau)|$, which describes the correlation of the speckle patterns separated by a certain time delay. In a statically ordered system, $|F(\tau)|$ will remain unchanged, while speckle dynamics causes it to drop as a function of the time delay. Distinct from $\mathrm{La}_{2-x} \mathrm{Ba}_{x} \mathrm{CuO}_{4}$ (LBCO), in which the $\mathrm{CO}$ is static over a timescale of at least $2 \mathrm{~h}[33,34]$, $|F(\tau)|$ in LSNO decays after several minutes for both CO and SO, indicating charge and spin dynamics (Fig. 3). Moreover, we find that $\mathrm{CO}$ and $\mathrm{SO}$ are both most stable around $70 \mathrm{~K}$ when they have longest correlation lengths, but $\mathrm{SO}$ is more stable than $\mathrm{CO}$ at $70 \mathrm{~K}$. Although stripes involve a comodulation of both charge and spin [30], we observe that these have different thermal evolution. As the temperature is driven away from $70 \mathrm{~K}$, the temporal stability for SO decreases faster, indicating that $\mathrm{SO}$ is less stable against temperature changes. A qualitatively, but not quantitatively, similar trend in SO was reported recently in Ref. [36]. The longer timescales observed here may reflect sample discrimination in strontium and oxygen compositions or improved coherent flux and stability at CSX compared to the Advanced Light Source.

From simple energetic considerations, if an order is less temporally stable and has shorter correlation lengths, one would expect it to be more fragile to thermal disturbance. 

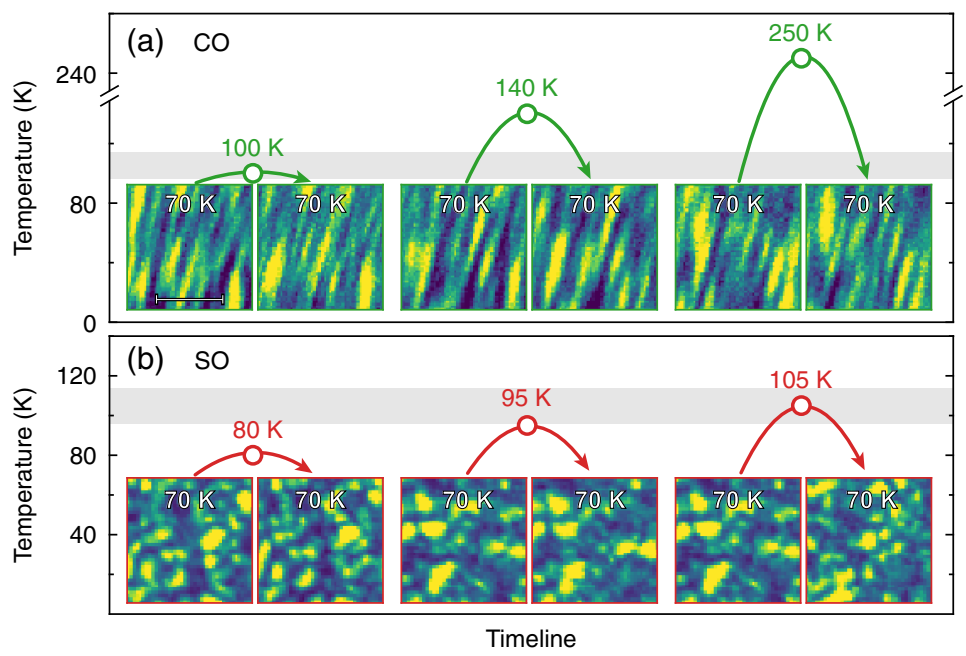

(c)

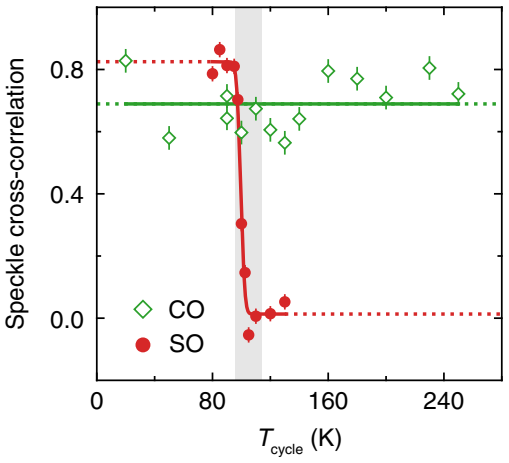

FIG. 4. Domain memory in CO but not SO. (a),(b) Representative speckle images before and after thermal cyclings, which are indicated by the curved arrows. The open circles stand for the cycling temperatures $T_{\text {cycle }}$. For each measurement, we collected images at $70 \mathrm{~K}$, changed the temperature to $T_{\text {cycle }}$, and waited for $10 \mathrm{~min}$. Then the sample was cooled back to $70 \mathrm{~K}$ and equilibrated for $30 \mathrm{~min}$ before collecting another image. For both the heating and cooling processes, the temperature ramping rate was fixed to $4 \mathrm{~K} / \mathrm{min}$. The white bar in the first speckle image indicates $10^{-3} \AA^{-1}$. (c) Temperature dependence of the normalized speckle cross-correlation function $\xi_{\mathrm{CC}}$. The solid and dashed lines are guides to the eye. The shaded area indicates the range of CO and SO transition temperatures.

The unexpected robustness of $\mathrm{CO}$ against temperature changes indicates that $\mathrm{CO}$ is coupled to other degrees of freedom which constrain the $\mathrm{CO}$ domains during and after the charge condensation (Fig. 3). Such hypotheses can be examined more deeply in terms of domain-pinning memory effects. Since the speckle positions are primarily determined by the positions of the ordering domains, the comparison of speckle patterns collected at $70 \mathrm{~K}$ before and after cycling the sample temperature to $T_{\text {cycle }}$ can evaluate whether the domain distributions are reproduced [35]. The usage of a $\mathrm{Pt}$ mask further ensures that the illuminated sample volume is fixed throughout the thermal cycling [Fig. 1(b)]. It turns out that the speckle patterns of $\mathrm{CO}$ are rather similar with $T_{\text {cycle }}$ up to $250 \mathrm{~K}$, well above $T_{\text {CO }}$ [Fig. 4(a)]. The SO speckles, however, change their positions once $T_{\text {cycle }}$ crosses $T_{\text {SO }}(\sim 100 \mathrm{~K})$ [Fig. 4(b)]. This effect can be quantified by calculating the normalized cross-correlation function $\xi_{\mathrm{CC}}$ which describes the similarity between two speckle patterns [35,39]. $\xi_{\mathrm{CC}}$ approaches zero when the two speckle images are different, while two identical images will give $\xi_{\mathrm{CC}}$ of one. Correspondingly, we calculate $\xi_{\mathrm{CC}}$ for both $\mathrm{CO}$ and $\mathrm{SO}$ speckle patterns with different $T_{\text {cycle }}$ [Fig. 4(c)]. The results again show that $\mathrm{CO}$ domain distributions are essentially unchanged after thermal cycling to a temperature far above $T_{\mathrm{CO}}$, while the $\mathrm{SO}$ speckle pattern loses reproducibility after the system is driven into the disordered state.

The domain memory effect of $\mathrm{CO}$ is caused by coupling to the host lattice. Local potentials arising from structural disorder induced, for example, by $\mathrm{Sr}$ doping, structural domain boundaries, or octahedral tilts provide nucleation centers for the $\mathrm{CO}$ domains and effectively pin the domains during stripe condensation. Since the average lattice structure of LSNO has translational symmetry over a length scale smaller than $\mathrm{CO}$ wavelength, it cannot, itself, pin the $\mathrm{CO}$ domains into reproducible locations. In charge-ordered cuprate $\mathrm{LBCO}$, the speckle pattern of $\mathrm{CO}$ domains loses memory after the sample is heated across the transition temperature from the low-temperature-orthorhombic (LTO) phase into the high-temperature-tetragonal (HTT) phase [35]. Thus, it is expected that the pinning landscape for $\mathrm{CO}$ in LBCO is constrained by twin boundaries created by the LTO structural distortion. In LSNO, the lattice remains in the HTT phase and no long-range LTO distortion is observed [53]. However, short-range stripe-related distortions have been reported to persist up to high temperatures [54]. It is possible that either these distortions or local defects due to Sr-related doping disorder determine the pinning landscape of LSNO in a similar manner.

The pinning effect of $\mathrm{CO}$ to the structural disorder also evinces the relevance of EPC in nickelates, which has been illustrated by the discovery of phonon anomalies and nematic behaviors in LSNO [55-58]. It has been argued theoretically that without EPC CO will remain dynamic and not order [32]. For structure-driven $\mathrm{CO}$, phonons soften to zero energy and drag the valence charge along with it to form spatial modulations. Here, however, phonons are softened by a maximum of $20 \%$ [56], and charge stripes are formed to reduce Coulomb interactions. EPC helps pin preformed charge stripes according to the lattice symmetry, promoting the static CO. The presence of EPC further couples the CO domains to structural disorder, which strengthens the $\mathrm{CO}$ against thermal fluctuations. Consequently, when $\mathrm{CO}$ and $\mathrm{SO}$ lose correlations 
progressively upon heating or cooling away from $70 \mathrm{~K}$, the fluctuations of $\mathrm{CO}$ speckles increase more slowly (Fig. 3).

SO behaves in a different way. During the formation of $\mathrm{SO}$, the spins can align either parallel or antiparallel to their quantization axis. This would disrupt the reproducibility of SO speckles after thermal cycling across $T_{\text {So }}$ even if the domain walls are in the same place (Fig. 4). Moreover, the rotational degree of freedom provides an additional fluctuation channel to the ordered spins, facilitating the loss of SO stability when driven away from $70 \mathrm{~K}$ (Fig. 3). This is in line with the observation of spin reorientation in LSNO at low temperatures $[24,27,38]$.

The robustness of $\mathrm{CO}$ stability and its pinning to the lattice demonstrate that the stripe order in LSNO is charge driven. This directly verifies prior theoretical predictions based on Landau theory of coupled charge and spin order parameters [30] and may reflect that stripe order is charge driven, in general. Our approach will be extendable to other materials and even to other degrees of freedom such as orbital order, bringing a powerful means to disentangle the formation mechanisms of intertwined ground states.

This material is based upon work supported by the U.S. Department of Energy (DOE), Office of Basic Energy Sciences. Work at Brookhaven National Laboratory was supported by the U.S. DOE, Office of Science, Office of Basic Energy Sciences, under Contract No. DESC0012704. The work at Argonne National Laboratory was supported by the U.S. Department of Energy, Office of Basic Energy Sciences, under Contract No. DEAC0206CH11357. D. G. M. acknowledges funding from the Swiss National Science Foundation, Fellowship No. P2EZP2_175092. This research used resources at the 23-ID-1 beam line of the National Synchrotron Light Source, a U.S. DOE Office of Science User Facility operated for the DOE Office of Science by Brookhaven National Laboratory under Contract No. DE-SC0012704.

*yshen@bnl.gov

mdean@bnl.gov

[1] A. V. Chumak, V. I. Vasyuchka, A. A. Serga, and B. Hillebrands, Magnon spintronics, Nat. Phys. 11, 453 (2015).

[2] E. Fradkin, S. A. Kivelson, and J. M. Tranquada, Theory of intertwined orders in high temperature superconductors, Rev. Mod. Phys. 87, 457 (2015).

[3] S. Mori, C. H. Chen, and S. W. Cheong, Pairing of chargeordered stripes in (La, Ca) $\mathrm{MnO}_{3}$, Nature (London) 392, 473 (1998).

[4] P. A. Lee, N. Nagaosa, and X.-G. Wen, Doping a Mott insulator: Physics of high-temperature superconductivity, Rev. Mod. Phys. 78, 17 (2006).

[5] H. Ulbrich and M. Braden, Neutron scattering studies on stripe phases in non-cuprate materials, Physica (Amsterdam) 481C, 31 (2012).
[6] R. Comin and A. Damascelli, Resonant x-ray scattering studies of charge order in cuprates, Annu. Rev. Condens. Matter Phys. 7, 369 (2016).

[7] J. M. Tranquada, B. J. Sternlieb, J. D. Axe, Y. Nakamura, and S. Uchida, Evidence for stripe correlations of spins and holes in copper oxide superconductors, Nature (London) 375, 561 (1995).

[8] H. A. Mook, P. Dai, F. Dogan, and R. D. Hunt, Onedimensional nature of the magnetic fluctuations in $\mathrm{YBa}_{2} \mathrm{Cu}_{3} \mathrm{O}_{6.6}$, Nature (London) 404, 729 (2000).

[9] J. E. Hoffman, E. W. Hudson, K. M. Lang, V. Madhavan, H. Eisaki, S. Uchida, and J. C. Davis, A four unit cell periodic pattern of quasi-particle states surrounding vortex cores in $\mathrm{Bi}_{2} \mathrm{Sr}_{2} \mathrm{CaCu}_{2} \mathrm{O}_{8+\delta}$, Science 295, 466 (2002).

[10] J. M. Tranquada, D. J. Buttrey, V. Sachan, and J. E. Lorenzo, Simultaneous Ordering of Holes and Spins in $\mathrm{La}_{2} \mathrm{NiO}_{4.125}$, Phys. Rev. Lett. 73, 1003 (1994).

[11] J. M. Tranquada, D. J. Buttrey, and V. Sachan, Incommensurate stripe order in $\mathrm{La}_{2-x} \mathrm{Sr}_{x} \mathrm{NiO}_{4}$ with $x=0.225$, Phys. Rev. B 54, 12318 (1996).

[12] D. Li, K. Lee, B. Y. Wang, M. Osada, S. Crossley, H. R. Lee, Y. Cui, Y. Hikita, and H. Y. Hwang, Superconductivity in an infinite-layer nickelate, Nature (London) 572, 624 (2019).

[13] J. Zhang, Y.-S. Chen, D. Phelan, H. Zheng, M. R. Norman, and J. F. Mitchell, Stacked charge stripes in the quasi-2D trilayer nickelate $\mathrm{La}_{4} \mathrm{Ni}_{3} \mathrm{O}_{8}$, Proc. Natl. Acad. Sci. U.S.A. 113, 8945 (2016).

[14] J. Zhang, A. S. Botana, J. W. Freeland, D. Phelan, H. Zheng, V. Pardo, M. R. Norman, and J. F. Mitchell, Large orbital polarization in a metallic square-planar nickelate, Nat. Phys. 13, 864 (2017).

[15] J. Zhang, D. M. Pajerowski, A. S. Botana, H. Zheng, L. Harriger, J. Rodriguez-Rivera, J. P. C. Ruff, N. J. Schreiber, B. Wang, Y.-S. Chen, W. C. Chen, M. R. Norman, S. Rosenkranz, J. F. Mitchell, and D. Phelan, Spin Stripe Order in a Square Planar Trilayer Nickelate, Phys. Rev. Lett. 122, 247201 (2019).

[16] J. Q. Lin et al., Strong Superexchange in a $d^{9-\delta}$ Nickelate Revealed by Resonant Inelastic X-Ray Scattering, Phys. Rev. Lett. 126, 087001 (2021).

[17] V. J. Emery, S. A. Kivelson, and O. Zachar, Spin-gap proximity effect mechanism of high-temperature superconductivity, Phys. Rev. B 56, 6120 (1997).

[18] S. A. Kivelson, E. Fradkin, and V. J. Emery, Electronic liquid-crystal phases of a doped mott insulator, Nature (London) 393, 550 (1998).

[19] D. F. Agterberg, J. C. Séamus Davis, S. D. Edkins, E. Fradkin, D. J. Van Harlingen, S. A. Kivelson, P. A. Lee, L. Radzihovsky, J. M. Tranquada, and Y. Wang, The physics of pair-density waves: Cuprate superconductors and beyond, Annu. Rev. Condens. Matter Phys. 11, 231 (2020).

[20] C. H. Chen, S. W. Cheong, and A.S. Cooper, Charge Modulations in $\mathrm{La}_{2-x} \mathrm{Sr}_{x} \mathrm{NiO}_{4+y}$ : Ordering of Polarons, Phys. Rev. Lett. 71, 2461 (1993).

[21] S. W. Cheong, H. Y. Hwang, C. H. Chen, B. Batlogg, L. W. Rupp, and S.A. Carter, Charge-ordered states in (La, $\mathrm{Sr})_{2} \mathrm{NiO}_{4}$ for hole concentrations $n_{h}=1 / 3$ and $1 / 2$, Phys. Rev. B 49, 7088 (1994). 
[22] S.H. Lee and S.W. Cheong, Melting of Quasi-TwoDimensional Charge Stripes in $\mathrm{La}_{5 / 3} \mathrm{Sr}_{1 / 3} \mathrm{NiO}_{4}$, Phys. Rev. Lett. 79, 2514 (1997).

[23] H. Yoshizawa, T. Kakeshita, R. Kajimoto, T. Tanabe, T. Katsufuji, and Y. Tokura, Stripe order at low temperatures in $\mathrm{La}_{2-x} \mathrm{Sr}_{x} \mathrm{NiO}_{4}$ with $0.289 \lesssim x \lesssim 0.5$, Phys. Rev. B 61, R854 (2000).

[24] S. H. Lee, S. W. Cheong, K. Yamada, and C. F. Majkrzak, Charge and canted spin order in $\mathrm{La}_{2-x} \mathrm{Sr}_{x} \mathrm{NiO}_{4}(x=0.275$ and 1/3), Phys. Rev. B 63, 060405(R) (2001).

[25] R. Kajimoto, T. Kakeshita, H. Yoshizawa, T. Tanabe, T. Katsufuji, and Y. Tokura, Hole concentration dependence of the ordering process of the stripe order in $\mathrm{La}_{2-x} \mathrm{Sr}_{x} \mathrm{NiO}_{4}$, Phys. Rev. B 64, 144432 (2001).

[26] M. E. Ghazi, P. D. Spencer, S. B. Wilkins, P. D. Hatton, D. Mannix, D. Prabhakaran, A. T. Boothroyd, and S.W. Cheong, Incommensurate charge stripe ordering in $\mathrm{La}_{2-x} \mathrm{Sr}_{x} \mathrm{NiO}_{4}$ for $x=(0.33,0.30,0.275)$, Phys. Rev. B 70, 144507 (2004).

[27] P. G. Freeman, A. T. Boothroyd, D. Prabhakaran, M. Enderle, and C. Niedermayer, Stripe order and magnetic transitions in $\mathrm{La}_{2-x} \mathrm{Sr}_{x} \mathrm{NiO}_{4}$, Phys. Rev. B 70, 024413 (2004).

[28] M. Raczkowski, R. Frésard, and A. M. Oleś, Microscopic origin of diagonal stripe phases in doped nickelates, Phys. Rev. B 73, 094429 (2006).

[29] P. Wochner, J. M. Tranquada, D. J. Buttrey, and V. Sachan, Neutron-diffraction study of stripe order in $\mathrm{La}_{2} \mathrm{NiO}_{4+\delta}$ with $\delta=2 / 15$, Phys. Rev. B 57, 1066 (1998).

[30] O. Zachar, S. A. Kivelson, and V. J. Emery, Landau theory of stripe phases in cuprates and nickelates, Phys. Rev. B 57, 1422 (1998).

[31] J. Zaanen and P. B. Littlewood, Freezing electronic correlations by polaronic instabilities in doped $\mathrm{La}_{2} \mathrm{NiO}_{4}$, Phys. Rev. B 50, 7222 (1994).

[32] T. Hotta and E. Dagotto, Orbital Ordering, New Phases, and Stripe Formation in Doped Layered Nickelates, Phys. Rev. Lett. 92, 227201 (2004).

[33] X. M. Chen, V. Thampy, C. Mazzoli, A. M. Barbour, H. Miao, G. D. Gu, Y. Cao, J. M. Tranquada, M. P. M. Dean, and S. B. Wilkins, Remarkable Stability of Charge Density Wave Order in $\mathrm{La}_{1.875} \mathrm{Ba}_{0.125} \mathrm{CuO}_{4}$, Phys. Rev. Lett. 117, 167001 (2016).

[34] V. Thampy, X. M. Chen, Y. Cao, C. Mazzoli, A. M. Barbour, W. Hu, H. Miao, G. Fabbris, R. D. Zhong, G. D. Gu, J. M. Tranquada, I. K. Robinson, S. B. Wilkins, and M. P. M. Dean, Static charge-density-wave order in the superconducting state of $\mathrm{La}_{2-x} \mathrm{Ba}_{x} \mathrm{CuO}_{4}$, Phys. Rev. B 95, 241111(R) (2017).

[35] X. M. Chen, C. Mazzoli, Y. Cao, V. Thampy, A. M. Barbour, W. Hu, M. Lu, T. A. Assefa, H. Miao, G. Fabbris, G. D. Gu, J. M. Tranquada, M. P. M. Dean, S. B. Wilkins, and I. K. Robinson, Charge density wave memory in a cuprate superconductor, Nat. Commun. 10, 1435 (2019).

[36] A. Ricci, N. Poccia, G. Campi, S. Mishra, L. Müller, B. Joseph, B. Shi, A. Zozulya, M. Buchholz, C. Trabant, J. C. T. Lee, J. Viefhaus, J. B. Goedkoop, A. A. Nugroho, M. Braden, S. Roy, M. Sprung, and C. Schüßler-Langeheine, Intrinsic spatial and temporal destabilization of incommensurate stripes at low temperatures, arXiv:1912.07306.
[37] D. Prabhakaran, P. Isla, and A. T. Boothroyd, Growth of large $\mathrm{La}_{2-x} \mathrm{Sr}_{x} \mathrm{NiO}_{4+\delta}$ single crystals by the floating-zone technique, J. Cryst. Growth 237-239, 815 (2002).

[38] P. G. Freeman, A. T. Boothroyd, D. Prabhakaran, and J. Lorenzana, Magnetization of $\mathrm{La}_{2-x} \mathrm{Sr}_{x} \mathrm{NiO}_{4+\delta} \quad(0 \ll$ $x \ll 0.5)$ : Spin-glass and memory effects, Phys. Rev. B 73, 014434 (2006).

[39] See Supplemental Material at http://link.aps.org/ supplemental/10.1103/PhysRevLett.126.177601 for details of the $\mathrm{x}$-ray measurements, sample preparation and the wave vectors of charge and spin order peaks.

[40] National Syncrotron light Source II Website, https://www .bnl.gov/ps/accelerator/, accessed: 2020-12-02.

[41] C. Schüssler-Langeheine, J. Schlappa, A. Tanaka, Z. Hu, C. F. Chang, E. Schierle, M. Benomar, H. Ott, E. Weschke, G. Kaindl, O. Friedt, G. A. Sawatzky, H.-J. Lin, C. T. Chen, M. Braden, and L. H. Tjeng, Spectroscopy of Stripe Order in $\mathrm{La}_{1.8} \mathrm{Sr}_{0.2} \mathrm{NiO}_{4}$ Using Resonant Soft X-Ray Diffraction, Phys. Rev. Lett. 95, 156402 (2005).

[42] J. Fink, E. Schierle, E. Weschke, and J Geck, Resonant elastic soft x-ray scattering, Rep. Prog. Phys. 76, 056502 (2013).

[43] R. Kukreja, N. Hua, J. Ruby, A. Barbour, W. Hu, C. Mazzoli, S. Wilkins, E. E. Fullerton, and O. G. Shpyrko, Orbital Domain Dynamics in Magnetite below the Verwey Transition, Phys. Rev. Lett. 121, 177601 (2018).

[44] $\xi=a / \sqrt{2} \Delta H$ where $\Delta H$ is half width at half maximum change in $\Delta H$ for a $[H, H, 0]$ scan.

[45] P. D. Hatton, M. E. Ghazi, S. B. Wilkins, P. D. Spencer, D. Mannix, T. d'Almeida, P. Prabhakaran, A. Boothroyd, and S. W. Cheong, X-ray scattering studies of charge stripes in $\mathrm{La}_{2-x} \mathrm{Sr}_{x} \mathrm{NiO}_{4} \quad(x=0.20-0.33)$, Physica (Amsterdam) 318B, 289 (2002).

[46] J. Schlappa, C. F. Chang, E. Schierle, A. Tanaka, R. Feyerherm, Z. Hu, H. Ott, O. Friedt, E. Dudzik, H. H. Hung, M. Benomar, M. Braden, L.H. Tjeng, and C. Schüßler-Langeheine, Static and fluctuating stripe order observed by resonant soft x-ray diffraction in $\mathrm{La}_{1.8} \mathrm{Sr}_{0.2} \mathrm{NiO}_{4}$, arXiv:0903.0994.

[47] V. Sachan, D. J. Buttrey, J. M. Tranquada, J. E. Lorenzo, and G. Shirane, Charge and spin ordering in $\mathrm{La}_{2-x} \mathrm{Sr}_{x} \mathrm{NiO}_{4.00}$ with $x=0.135$ and 0.20, Phys. Rev. B 51, 12742 (1995).

[48] K. Ishizaka, T. Arima, Y. Murakami, R. Kajimoto, H. Yoshizawa, N. Nagaosa, and Y. Tokura, CommensurateIncommensurate Crossover of Charge Stripe in $\mathrm{La}_{2-x} \mathrm{Sr}_{x} \mathrm{NiO}_{4}(x \sim 1 / 3)$, Phys. Rev. Lett. 92, 196404 (2004).

[49] H. Miao, R. Fumagalli, M. Rossi, J. Lorenzana, G. Seibold, F. Yakhou-Harris, K. Kummer, N. B. Brookes, G. D. Gu, L. Braicovich, G. Ghiringhelli, and M. P. M. Dean, Formation of Incommensurate Charge Density Waves in Cuprates, Phys. Rev. X 9, 031042 (2019).

[50] S. Brauer, G. B. Stephenson, M. Sutton, R. Brüning, E. Dufresne, S. G. J. Mochrie, G. Grübel, J. Als-Nielsen, and D. L. Abernathy, X-Ray Intensity Fluctuation Spectroscopy Observations of Critical Dynamics in $\mathrm{Fe}_{3} \mathrm{Al}$, Phys. Rev. Lett. 74, 2010 (1995).

[51] O. Shpyrko, X-ray photon correlation spectroscopy, J. Synchrotron Radiat. 21, 1057 (2014).

[52] S. Lee, J. Jiang, G. Fabbris, A. S. Disa, M. P. M. Dean, S. Ismail-Beigi, F. J. Walker, C. Mazzoli, and C. H. Ahn, 
Dimensionality-driven antiferromagnetic dynamics in nickelate heterostructures (to be published).

[53] M. Hücker, K. Chung, M. Chand, T. Vogt, J. M. Tranquada, and D. J. Buttrey, Oxygen and strontium codoping of $\mathrm{La}_{2} \mathrm{NiO}_{4}$ : Room-temperature phase diagrams, Phys. Rev. B 70, 064105 (2004).

[54] A. M. Milinda Abeykoon, E. S. Božin, W.-G. Yin, G. Gu, J. P. Hill, J. M. Tranquada, and S. J. L. Billinge, Evidence for Short-Range-Ordered Charge Stripes far above the ChargeOrdering Transition in $\mathrm{La}_{1.67} \mathrm{Sr}_{0.33} \mathrm{NiO}_{4}$, Phys. Rev. Lett. 111, 096404 (2013).

[55] Yu. G. Pashkevich, V. A. Blinkin, V. P. Gnezdilov, V. V. Tsapenko, V. V. Eremenko, P. Lemmens, M. Fischer, M. Grove, G. Güntherodt, L. Degiorgi, P. Wachter, J. M.
Tranquada, and D. J. Buttrey, Stripe Conductivity in $\mathrm{La}_{1.775} \mathrm{Sr}_{0.225} \mathrm{NiO}_{4}$, Phys. Rev. Lett. 84, 3919 (2000).

[56] J. M. Tranquada, K. Nakajima, M. Braden, L. Pintschovius, and R. J. McQueeney, Bond-Stretching-Phonon Anomalies in Stripe-Ordered $\mathrm{La}_{1.69} \mathrm{Sr}_{0.31} \mathrm{NiO}_{4}$, Phys. Rev. Lett. 88, 075505 (2002).

[57] A. M. Merritt, A. D. Christianson, A. Banerjee, G. D. Gu, A. S. Mishchenko, and D. Reznik, Giant electron-phonon coupling of the breathing plane oxygen phonons in the dynamic stripe phase of $\mathrm{La}_{1.67} \mathrm{Sr}_{0.33} \mathrm{NiO}_{4}$, Sci. Rep. 10, 11426 (2020).

[58] R. Zhong, B. L. Winn, G. Gu, D. Reznik, and J. M. Tranquada, Evidence for a Nematic Phase in $\mathrm{La}_{1.75} \mathrm{Sr}_{0.25} \mathrm{NiO}_{4}$, Phys. Rev. Lett. 118, 177601 (2017). 